\title{
Quantum Interference without Quantum Mechanics
}

\author{
Arend Niehaus* \\ Utrecht University, Utrecht, The Netherlands \\ Email: arendniehaus@aol.com
}

How to cite this paper: Niehaus, A. (2019) Quantum Interference without Quantum Mechanics. Journal of Modern Physics, 10, 423-431.

https://doi.org/10.4236/jmp.2019.104027

Received: February 9, 2019

Accepted: March 16, 2019

Published: March 19, 2019

Copyright $\odot 2019$ by author(s) and Scientific Research Publishing Inc. This work is licensed under the Creative Commons Attribution International License (CC BY 4.0).

http://creativecommons.org/licenses/by/4.0/

\begin{abstract}
A recently proposed model of the Dirac electron, which has been shown to describe several observed properties of the particle correctly, is in the present paper shown to be also able to explain quantum interference by classical probabilities. According to this model, the electron is not point-like, but rather an "entity with structure", formed by a fast periodic motion of a "light-like object", whose momentum $(p)$ causes the angular momentum responsible for the spin, and whose energy $(E=p c)$ is equal to the energy of the electron, $\mathrm{mc}^{2}$. A qualitative description of the model is given, together with the quantitative formulae that allow to discuss interference. Applied to the experimental situation of the "two-slit" experiment, the formulae yield the same time dependence of the detection probability as the quantum mechanical treatment, and hence the same interference pattern. In contrast to quantum mechanics, the pattern is due to "particle interference" rather than to "wave interference". No wave-particle paradox arises. The merits of the model are summarized, and its physical content discussed.
\end{abstract}

\section{Keywords}

Interpretation of Quantum Mechanics, Quantum Interference, Classical Probability

\section{Introduction}

Quantum mechanics treats the electron as a point-like particle having a certain mass, but no structure. To describe observed phenomena correctly, it ascribes certain other properties to the particle, for instance spin, and a wavelike nature, which are "non-classical", and cannot be "derived" (e.g. [1]). The validity of the theory is unquestioned; however, its interpretation is still subject of debate (see, f.i., [2]), because the "quantum world" it creates contains numerous well known paradoxes.

${ }^{*}$ Retired Professor of Physics. 
There have been attempts to escape interpretational problems by supposing that the electron, and possibly other elementary particles also, do have an internal structure that possibly could explain their properties. Especially, the fact that a so called "Zitterbewegung (ZBW)" [3] is one of the properties arising from the relativistic quantum theoretical treatment of the free electron, has led to the proposal of a dynamic substructure [4] [5] [6]. Typical time scale for such a structure would be the very short period $(\tau)$ of the $(\mathrm{ZBW})$ :

$$
\tau=2 \pi / \omega_{Z B W} \text { with } \omega_{Z B W}=2 c / L_{0} \text {, leading to } \tau=2 \pi L_{0} / 2 c \approx 4 \cdot 10^{-21} \mathrm{~s} \text {. }
$$

Theoretical analyses have indeed shown that the spin, arising in the Dirac theory, can be related to a motion; however, an extended (ZBW), not predicted by the Dirac theory, would be necessary to explain the properties of the electron [6]. Also models of the electron, based on the (ZBW), have been proposed [7] [8]. For a recent discussion we refer to [9].

The model to be used in this paper, is also based on the assumption of a $(\mathrm{ZBW})$. But the new aspect is that, the (ZBW) is not ascribed to the electron, but rather to a light-like object that possesses momentum and energy, and establishes the electron by its periodic motion around a point in space. The model has been developed in two recent publications [10] [11] ${ }^{1}$ of the present author, and has been shown to explain the properties like spin, magnetic moment, and mass, of the electron. In the present paper it will be shown to be also able to explain quantum interference using classical probabilities. First, we give a short qualitative description of the model.

Starting from the assumption that spin is caused by orbital motion due to an extended (ZBW), a probability distribution of orientation and value of an instantaneous orbital angular momentum is designed, which describes spin and spin measurements in accordance with experiment. Under the assumption that the "object" that causes the angular momenta is "light-like", with momentum ( $p$ $=\hbar / L$ ), probability distributions for orientation and length of the instantaneous position vector of the "light-like object", which we will call "quantum", are derived from the angular distributions. The instantaneous positions of the quantum turn out to lie on a torus around a fixed point, the torus radius $\left(R_{t}\right)$ being equal to the radius of the circle $\left(R_{c}\right)$ the torus axis forms around the fixed point: $R_{t}=R_{c}=(L / 2)$. This special Torus is a so called Clifford Torus. The relation between instantaneous position vector $(r)$ of the quantum, its instantaneous momentum $(p)$, and the resulting instantaneous angular momentum vector $(I=r \times$ $p$ ) is depicted in Figure 1.

The Torus, on whose surface the instantaneous positions of the quantum are located, is shown in Figure 2.

In Cartesian coordinates the position vector is given by

$$
r(x, y, z)=L\left\{\cos ^{2}(\theta) \cos (\varphi), \cos ^{2}(\theta) \sin (\varphi), \sin (\theta) \cos (\theta)\right\}
$$

The idea is now, to describe the distributions in terms of the extended ZBW of

${ }^{1}$ Erroneously, the labels (a) and (b) of the two figures in Figure 3 were interchanged in the final typesetting. 


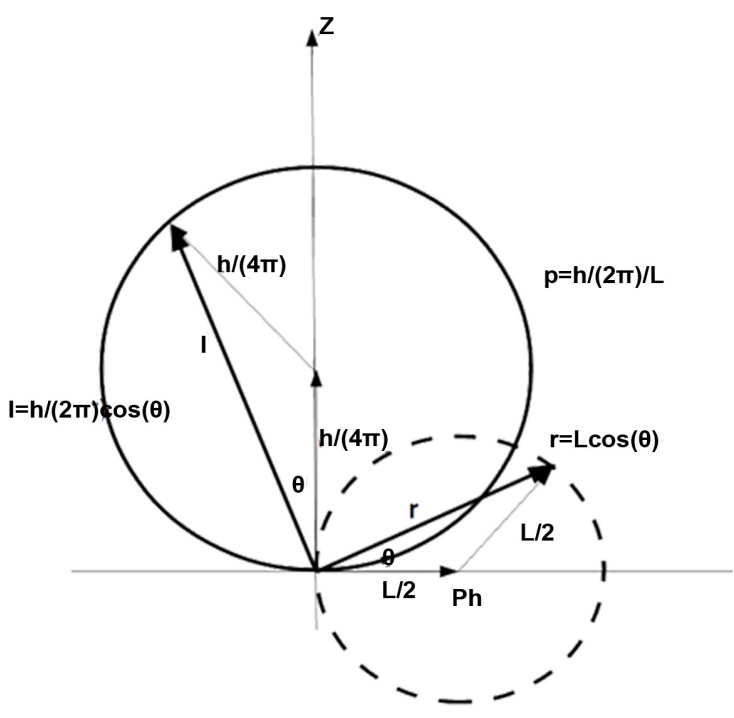

momentum $\mathbf{p}$ perpendicular w.r.t plane containig $\mathbf{r}$

Figure 1. Relation between the following instantaneous vectors, 1) momentum ( $p$ ) of the quantum, 2) its position $(\boldsymbol{r})$, and 3) of the resulting angular momentum $\boldsymbol{I}=\boldsymbol{r} \times \boldsymbol{p}$. Equal population of the circle around the point $\mathrm{Ph}$ by the quantum, together with cylindrical symmetry around the $\mathrm{Z}$-axis, lead to the required angular distribution $I(\theta)=$ $(h / 2 \pi) \cos (\theta)$, which explains spin and spin measurements correctly (see Ref. [10]).

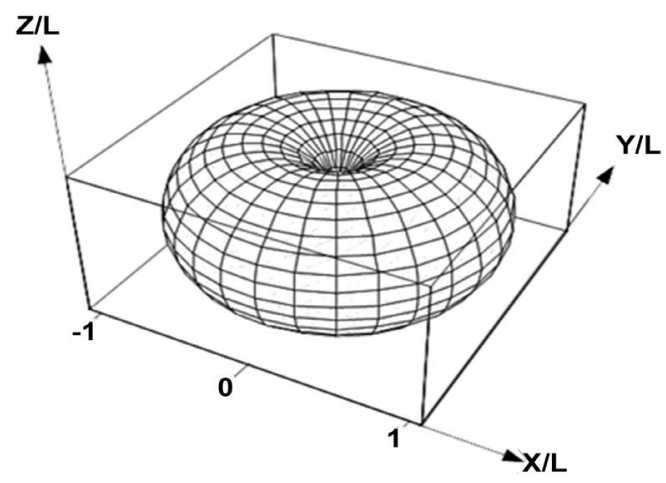

Figure 2. The instantaneous positions of the quantum are located on the surface of a Clifford Torus. The torus radius $\left(R_{t}\right)$ is equal to the radius of the circle $\left(R_{c}\right)$ the torus axis forms around the fixed point: $R_{t}=R_{c}=L / 2$.

the quantum. To obtain a motion of the quantum on the torus surface, we relate the angles $(\theta, \phi)$ as

$$
\varphi=\omega_{p} t ; \theta=\left(\frac{\omega_{t}}{2}\right) t ; \omega_{p}=n \omega_{t}
$$

The parameter $(t)$ is taken to be proper time, so that $\left(\omega_{p}, \omega_{t}\right)$ are frequencies, and the position vector describes a path in space and time on the surface of the 
torus. From (1) we get:

$$
r(x, y, z, t)=L\left\{\cos ^{2}\left(\left(\frac{\omega_{t}}{2}\right) t\right) \cos \left(\omega_{p} t\right), \cos ^{2}\left(\left(\frac{\omega_{t}}{2}\right) t\right) \sin \left(\omega_{p} t\right), \frac{\sin \left(\omega_{t} t\right)}{2}\right\}
$$

As an example, we show in Figure 3 the path for the case $n=10$ as calculated with (3).

Properties of the "entity" obtained using relation (3) as averages over an observation time, approach definite values after times sufficiently long compared to the period $\tau=4 \pi / \omega_{t}$. If $(L)$ is taken to be the reduced Compton wavelength $L$ $=\hbar / m c$, with $(m)$ the relativistic mass of the particle described, such values are, for instance, spin $=\hbar / 2$, and the correct magnetic moment $=\hbar e /\left(2 m_{0}\right)$. We stress that the value of the number $(n)$ has no influence on the general situation described so far.

We identify the "entity", if it is observed with a sufficiently low time-resolution, with the particle described. Then, the center of the torus is the position of the particle, and due to the dynamical origin of its torus-structure, it has its established properties.

In this paper we attempt to describe the property "wave nature" of moving particles. It becomes observable by the phenomenon interference, for instance in the two-slit experiment. Therefore, the velocity $(v)$ is introduced into the model represented so far by relation (3). Assuming a velocity in Z-direction, this is done in the following way:

1) The coordinate $(z)$ is replaced by $(z+v t) ; 2) \quad L=L_{0}\left(1-\beta^{2}\right)^{1 / 2}$ with $\beta=v / c$;3) $\omega_{p}=2 c / L_{0}$ so that $\omega_{p}$ becomes the ZBW-frequency arising in the Dirac theory.

The velocity component at the torus axis (at point $\mathrm{Ph}$ in Figure 1) in the $\mathrm{X}-\mathrm{Y}$ plane thus becomes $\omega_{p} L / 2=c\left(1-\beta^{2}\right)^{1 / 2}$, and the velocity component in $\mathrm{Z}$-direction is $(\beta c)$, resulting in a constant tangential speed (c) at the torus axis,

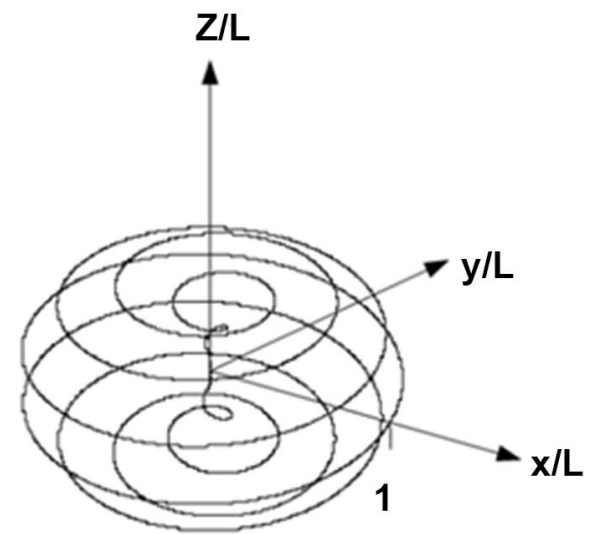

Figure 3. The closed path of the quantum. The "entity" it forms is taken to represent the electron for the case $\left(\omega_{p} / \omega_{t}=n=10\right)$. Coordinates are given in units of the reduced Compton wave length $(L)$. The path lies on the surface of the Torus shown in Figure 2. 
independent of $(v)$, as required. The ratio of the frequencies becomes: $\omega_{p} / \omega_{t}=n=\left(1-\beta^{2}\right)^{1 / 2} / \beta$, so that $\beta=1 /\left(1+n^{2}\right)^{1 / 2}$.

The complete model for the case of a velocity $(v=\beta c)$ in Z-direction may thus be represented by the modified relation (3) as follows:

$$
\begin{gathered}
r(x, y, z, t, \beta) \\
=L_{0} n \beta\left\{\cos ^{2}\left(\left(\frac{\omega_{t}}{2}\right) t\right) \cos \left(\omega_{p} t\right), \cos ^{2}\left(\left(\frac{\omega_{t}}{2}\right) t\right) \sin \left(\omega_{p} t\right), \frac{\sin \left(\omega_{t} t\right)}{2}+\frac{c t}{n L_{0}}\right\} \\
L_{0}=\frac{\hbar}{m_{0} c} ; \omega_{p}=\frac{2 c}{L_{0}} ; \omega_{t}=\frac{\omega_{p}}{n} ; n=\left(1-\beta^{2}\right)^{1 / 2} / \beta ;
\end{gathered}
$$

The model is completely general and contains no free parameters. In this paper we will demonstrate, in which way it describes observed interference phenomena of electrons correctly. As example we discuss the two slit experiment in paragraph (2).

\section{Interference}

First we show in Figure 4 a 3D-parametric plot of the path of the quantum during a time span of 2.5 periods $\left(\tau=4 \pi / \omega_{t}\right)$ for the case $n=10$, as calculated using relation (4).

In order to treat interference in the two-slit experiment, we need the velocity of the quantum in Z-direction ( $v q)$. As calculated using (4), this velocity is given by

$$
v q=2 v \cos ^{2}\left(\frac{\omega_{t}}{2}\right)=2 v \cos ^{2}\left(\omega_{D B} t\right)
$$

where, according to the definition of the Torus frequency given in (4), $\omega_{D B}$ is the De Broglie frequency defined as $\omega_{D B}=c / L_{D B}=c \mathrm{mv} / \hbar$.

For the case of many particles starting with a different phase $(\varphi)$ from a certain position in Z-direction, there arises a constant current of quanta in $Z$-direction, which is obtained by integration over $(\varphi)$. Assuming equal probability $(1 / 2 \pi)$ for different phases, the current becomes

$$
I(t)=\left(\frac{1}{2 \pi}\right) \int_{0}^{2 \pi} 2 v \cos ^{2}\left(\omega_{D B} t+\varphi\right) \mathrm{d} \varphi=v
$$

Let us now consider the normal two-slit situation in the context of the model, where diffracted particles from the two narrow slits separated by distance $(B)$ form currents into certain directions behind the slits, and hit the surface of a screen that is oriented perpendicular to the currents and positioned at a distance large compared to the distance between the two slits (see Figure 5).

Then, at any position on the screen determined by the diffraction angle $(+\delta)$, particles from both slits will arrive. For symmetry reasons, the same current will arrive at the angle $(-\delta)$. For each particle detected at $(+\delta)$ on the screen at time $(t)$, there is a certain probability that it originates from slit 1 , or slit 2 . According to classical probability theory [12], this conditional probability is obtained by the unification of the probabilities for the two slits, P1 and P2. 


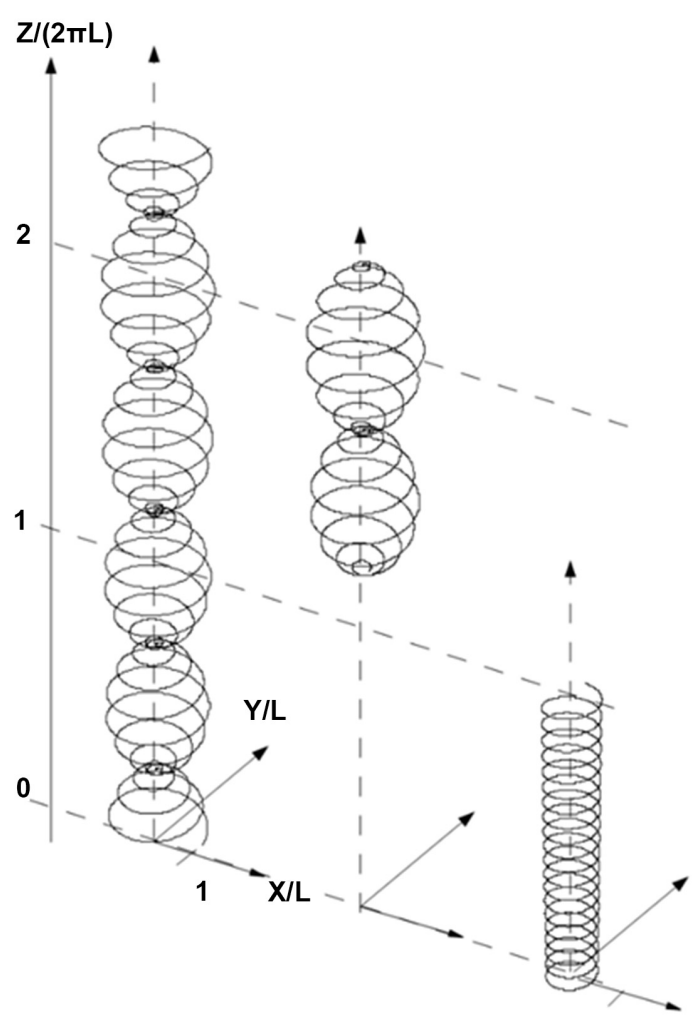

Figure 4. Traces from left to right: 1), A parametric 3D plot of the positions in real space of the quantum (in units of $L$ ) during the first 2.5 periods of the (EZBW), for the case $n=10.2)$, Path of the quantum during one period, starting 1.3 periods after $(t=0)$. 3), Path of the point $(\mathbf{P h})$ on the torus axis (see Figure 1 ) during the first period. Its tangential speed is (c) (see text). Progress of the "entity" in $z$-direction is seen to proceed at the velocity $v=2 \pi L / \tau=\beta c$, while progress of the quantum is given by $v q=2 v \cos ^{2}\left(\left(\omega_{t} / 2\right) t\right)$ (see text).

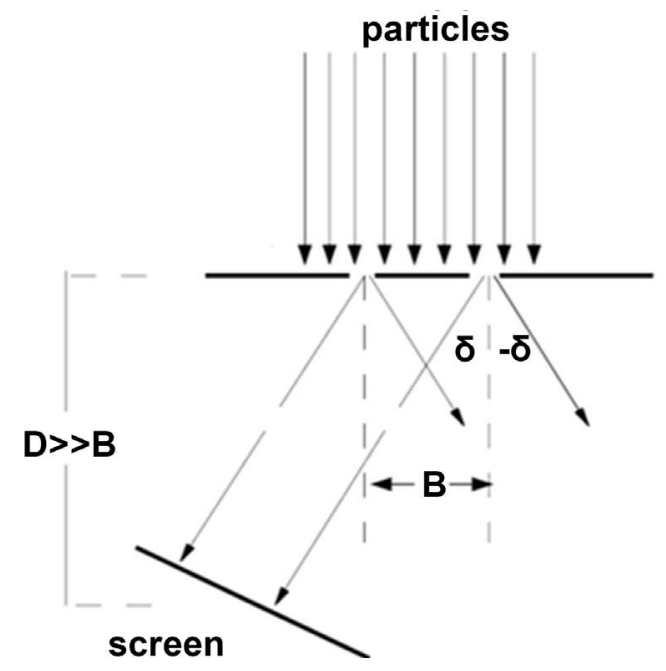

Figure 5. The two slit situation. The distance $(D)$ of the screen from the slits is large compared to the distance $(B)$ between the slits. 


$$
P(t, \varphi,+\delta)=(P 1 \text { or } P 2)=P 1+P 2-P 1 \cap P 2
$$

The last term in (7) is the intersection between the probabilities $P 1$ and $P 2$. Using relation (5), and setting the constant current to $V=1$, we have $P 1=2 \cos ^{2}\left(\omega_{D B} t+\varphi\right)$ and $P 2=2 \cos ^{2}\left(\omega_{D B}(t+D t)+\varphi\right)$. The intersection is the probability that both paths-via slit 1 , and via slit two-lead to detection at $(-\delta)$. In the present case, we identify the intersection as $P 1 \cap P 2=\left(\cos \left(\omega_{D B} t+\varphi\right)-\cos \left(\omega_{D B}(t+D t)\right)+\varphi\right)^{2}$. The total probability on the screen at position $(+\delta)$ is then obtained by integration of $(7)$ over possible initial conditions $(\varphi)$ :

$$
\begin{aligned}
P(t,+\delta) & \int_{0}^{2 \pi}\left\{2 \cos ^{2}\left(\omega_{D B} t+\varphi\right)+2 \cos ^{2}\left(\omega_{D B}(t+D t)+\varphi\right)\right. \\
& \left.-\left(\cos \left(\omega_{D B} t+\varphi\right)-\cos \left(\omega_{D B}(t+D t)+\varphi\right)\right)^{2}\right\} \mathrm{d} \varphi \\
= & 2 \cos ^{2}\left(\frac{\omega_{D B} D t}{2}\right)
\end{aligned}
$$

As is well known, the probability given by (8), is identical to the probability calculated in quantum mechanics for the superposition $(\Psi)$ of two equal amplitude, single momentum, particle De Broglie waves, starting from the two slits. This is indicated below.

$$
\Psi=(1 / \sqrt{2})\left(\mathrm{e}^{i k r}+\mathrm{e}^{i k^{\prime} r}\right) ; \Psi \Psi^{*}=2 \cos ^{2}\left(\frac{k\left(r-r^{\prime}\right)}{2}\right)=2 \cos ^{2}\left(\frac{\omega_{D B} D t}{2}\right)
$$

In (9) we used the De Broglie wavenumber $k=1 / L_{D B}=\omega_{D B} / c$, and the fact that $\left(r-r^{\prime}\right)=c D t$, the extra distance to be travelled from the more distant of the two slits.

The different derivations, (8) and (9), of the same result, clearly show the relation between model and quantum mechanics for the case of interference. Most remarkably, the non-local wave nature, ascribed in the quantum mechanical description to a single particle, the model ascribes to an ensemble of localized particles having a certain structure. Therefore, the paradoxical wave-particle dualism does not arise.

Below we demonstrate the prediction of a two-slit interference pattern using result (8) for a concrete case. The dependence of the detection probability on the angle $(\delta)$, for a given $V=\beta c$, is given through the relations $S=c D t=B \sin \delta$, and $L_{D B}=L / \beta=L_{0} \sqrt{ }\left(1-\beta^{2}\right) / \beta=L_{0} n$, so that the phase in (8) becomes $S / 2 L_{D B}=B \sin \delta / 2 n L_{0}=\pi\left(B / \lambda_{D B}\right) \sin \delta$.

Relation (8) is the predicted interference pattern for the case of two infinitely narrow slits separated by a distance $(B)$. In a realistic case, the slits have a finite width $(d)$, so that interference of particles coming from different positions within one slit arises, and leads to a certain single-slit interference pattern, which can be calculated approximately with relation (8) by replacing $(B)$ by $(d / 2)$. At sufficiently narrow slit widths $(d)$, and a slit distance $(B)$ considerably larger than $(d)$, the complete interference pattern thus becomes a modulation of the diffraction 
profile by the two-slit interference. In Figure 6 we show an example using $B / \lambda_{D B}$ $=5$ to calculate the two slit modulation, and $d=\lambda_{D B}$ to calculate the diffraction profile. At this width we have $d=\Delta x=\lambda_{D B}$, and a width of the diffraction peak corresponding to $\Delta p=p=\hbar / \lambda_{D \mathrm{~B}}$, so that the uncertainty relation inherent in the model becomes $\Delta p \Delta x=\hbar$.

As the main result of the present paper we state that the two-slit quantum interference is correctly described without quantum mechanics.

\section{Conclusion and Discussion}

The dynamic substructure, which the model ascribes to the particle, explains the two slit interference, one of the main quantum phenomena, without using quantum mechanics. In a similar way, energy quantization of bound states, as well as angular momentum quantization, is predicted by the model in agreement with observation. In addition, as has been shown in the previous papers [10] [11], the properties of the electron, mass, spin, and magnetic moment, follow from the substructure implied by the model. Further, the paradox of "wave-particle dualism" does not arise, because "wave interference" is explained as "particle interference".

As already mentioned, the model is based on the idea that spin might be explained as angular momentum caused by a quantum of momentum $(m c)$ when it passes a fixed point in space. The completed model of an elementary particle, used in this paper, may be visualized in a very simple way: The point $(\mathrm{Ph})$ (see Figure 1), which is located in the center of the circle containing the quantum, is identified as a photon, and the point, which is located in the center of this circulating photon, is identified as the particle. As shown in Figure 4, the photon forms a spiraling trace with tangential speed $(c)$, independent of the relative motion $(v)$ with respect to an observer, and its energy is $p c=m c^{2}$, with $\left(m=m_{0} / \sqrt{ }\left(1-\beta^{2}\right)\right)$. The momentum component of the photon in direction of relative motion, $\left(m v=m \beta c=m_{0} c / n\right)$, is the momentum of the "particle".

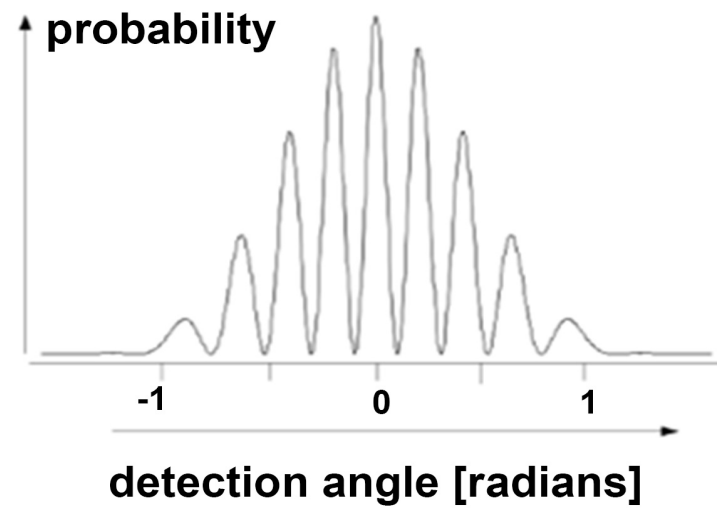

Figure 6. The two slit interference pattern for "particles" calculated with formula (8).The central diffraction peak, common to both slits, is modulated by the two-slit interference. The same interference pattern would be calculated using quantum mechanics. 
There remain important questions regarding the validity of the model. On the other hand, in our opinion, its demonstrated successes are too manifold and substantial to be accidental. Therefore, a thorough theoretical investigation of the model, especially of its relation to quantum field theory, seems interesting.

\section{Conflicts of Interest}

The author declares no conflicts of interest regarding the publication of this paper.

\section{References}

[1] Messiah, A. (1964) Quantum Mechanics. Vol. II, North Holland Publishing Company, Amsterdam, 540.

[2] Khrennikov, A. (2017) Foundations of Physics, 47, 1077-1099. https://doi.org/10.1007/s10701-017-0089-0

[3] Schroedinger, E. (1930) Sitzungsber. Preuss. Akad. Wiss. Phys.-Math. Kl., 24, 418.

[4] Hestenes, D. (1979) American Journal of Physics, 47, 399-415. https://doi.org/10.1119/1.11806

[5] Hestenes, D. (1990) Foundations of Physics, 20, 1213-1232. https://doi.org/10.1007/BF01889466

[6] Hestenes, D. (2003) Annales de la Fondation Louis de Broglie, 28, 390-408.

[7] Barut, A.O. and Sanghi, N. (1984) Physical Review Letters, 52, 2009-2012. https://doi.org/10.1103/PhysRevLett.52.2009

[8] Vaz Jr., J. (1995) Physics Letters B, 344, 149-157. https://doi.org/10.1016/0370-2693(94)01548-Q

[9] Pavsic, M., Recami, E., Waldyr, A., Rodriges Jr., G., Maccarrone, D., Racciti, F. and Salesi, G. (1993) Physics Letters B, 318, 481-488.

https://doi.org/10.1016/0370-2693(93)91543-V

[10] Niehaus, A. (2016) Foundations of Physics, 46, 3-13. https://doi.org/10.1007/s10701-015-9953-y

[11] Niehaus, A. (2017) Journal of Modern Physics, 8, 511-521. https://doi.org/10.4236/jmp.2017.84033

[12] Bauer, H. (2002) Wahrscheinlichkeitstheorie. De Gruyter, Berlin, New York. 